Tecno Lógicas

ISSN 0123-7799

Vol. 18, No. 35, pp. 11-20

Julio-diciembre de 2015

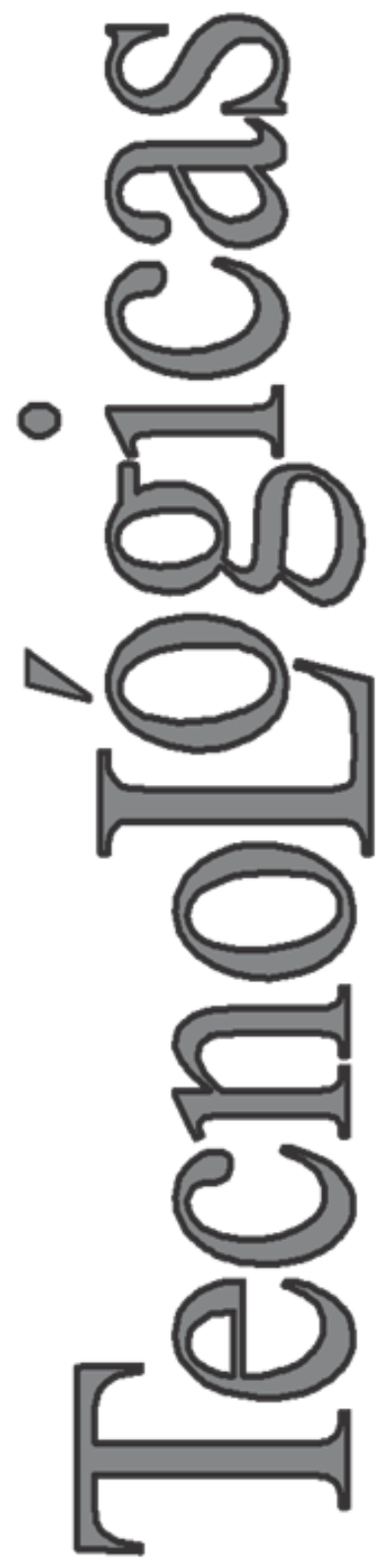

(C) Copyright 2015 por autores y Tecno Lógicas Este trabajo está licenciado bajo una Licencia Internacional Creative Commons Atribución (CC BY)

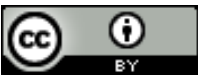

\section{Efecto del campo magnético estático homogéneo en la germinación y absorción de agua en semillas de soja}

\section{Static homogeneous magnetic field effects on germination and water absorption in soybean seeds}

\author{
Javier I. Torres-Osorio ${ }^{1}$, Jainer E. Aranzazu-Osorio ${ }^{2}$ \\ y María V. Carbonell-Padrino ${ }^{3}$
}

Recibido: 25 de junio de 2014, Aceptado: 19 de mayo de 2015

Cómo citar / How to cite

J. I. Torres-Osorio, J. E. Aranzazu-Osorio y M. V. Carbonell-Padrino, "Efecto del campo magnético estático homogéneo en la germinación y absorción de agua en semillas de soja”, Tecno Lógicas, vol. 18, no. 35, pp. 11-20, 2015.

1 M.Sc. en Instrumentación Física, Grupo de Investigación en Campos Electromagnéticos, Medio Ambiente y Salud Pública (línea magnetobiología), Departamento de Física, Universidad de Caldas, Manizales-Colombia, javier.torres@ucaldas.edu.co

2 Biólogo, joven investigador 2013-2015, Grupo de Investigación en Campos Electromagnéticos, Medio Ambiente y Salud Pública (línea magnetobiología), Universidad de Caldas, Manizales-Colombia, jearanzazuo@unal.edu.co

3 Ph. D. Ingeniero Agrónomo, Grupo de Investigación en Bioelectromagnetismo Aplicado a la Ingeniería Agroforestal, Escuela Técnica Superior de Ingenieros Agrónomos, Universidad Politécnica de Madrid, Madrid-España, victoria.carbonell@upm.es 


\section{Resumen}

Se presentan los resultados del estudio del efecto en la germinación y la absorción de agua en semillas de soja expuestas a campo magnético estático homogéneo de 125 y 300 mT durante 10, 20 y 60 minutos, usando como fuentes magnéticas un electroimán con núcleos cilíndricos (homogeneidad del 98\%) y un conjunto de imanes toroidales (homogeneidad del 88\%). Se realizaron dos experimentos factoriales con cuatro repeticiones de veinticinco semillas por tratamiento; un experimento con generación activa de campo magnético (electroimán) y otro con generación pasiva (imanes). Los resultados mostraron que hay mejor respuesta al usar el electroimán que ofrece mayor homogeneidad. En la germinación se determinó que el tiempo medio de germinación (TMG) y los tiempos para que germinaran el 10\%, 25\%, 50\% y $75 \%$ de las semillas presentaron diferencias significativas respecto al control, disminuyendo estos tiempos entre $22,89 \%$ y $5,09 \%$ para los tratamientos de $125 \mathrm{mT}-10 \mathrm{~min}$ y $300 \mathrm{mT}-10 \mathrm{~min}$. En el estudio de la absorción de agua se observó que en los tratamientos que expresaron mejor respuesta en la germinación se presenta una tendencia a incrementar esta capacidad durante las primeras 6 horas después de la siembra, sin embargo, no se registraron diferencias significativas para la absorción con respecto al control.

\section{Palabras clave}

Germinación, absorción de agua, estimulación magnética, soja, tiempo medio de germinación (TMG).

\section{Abstract}

We present the results of the study of the effect on germination and seed water absorption on soybean exposed to homogeneous magnetic field of 125 and $300 \mathrm{mT}$ during 10, 20, and 60 minutes, using as magnetic sources an electromagnet with cylindrical cores (homogeneity of 98\%) and a set of toroidal magnets (homogeneity of $88 \%$ ). The results showed better response in using the electromagnet that provides greater homogeneity. In the germination was determined that the mean germination time (MGT) and times necessary to germinate $10 \%, 25 \%, 50 \%$, and $75 \%$ significantly decreased relative to control, for treatments $125 \mathrm{mT}-10 \mathrm{~min}$ and $300 \mathrm{mT}-10 \mathrm{~min}$. For the water absorption was observed that in treatments expressed better response in germination show a tendency to increase the capacity of water absorption during the first 6 hours after seeding, however no significant differences in the absorption with respect to the control were recorded.

\section{Keywords}

Germination, water absorption, magnetic treatment, soybean, mean germination time (MGT). 


\section{INTRODUCCIÓN}

La idea aceptada que propone sensibilidad de algunos animales, insectos, bacterias y plantas al campo geomagnético [1] ha llevado a la investigación del efecto de dosis de exposición magnética bajo condiciones controladas en laboratorio que pueden afectar a diferentes sistemas biológicos. De los organismos investigados, las plantas conforman un grupo significativo por su importancia ambiental, económica y social; implementándose sobre ellas métodos físicos de tratamiento como: estimulación electromagnética $\mathrm{AC}$ sinusoidal $\mathrm{O}$ pulsada y estimulación magnética DC/AC, sinusoidal o pulsada. Han sido estudiadas varias especies agrícolas, entre las más reportadas: maíz [2]-[10], tomate [11]-[15], trigo [16]-[19], arveja [20]-[23] y soja [24][27] principalmente.

Para identificar el impacto de la estimulación magnética en las semillas, se analizan diversos parámetros biológicos como: el porcentaje de germinación, el tiempo medio de germinación (TMG), la longitud de la plántula, raíz y tallo, la masa fresca y seca de raíz [9], [15], [28], [29]; variables biofísicas como: conductividad, electroconductividad específica $\mathrm{y}$, absorción y adsorción de agua [4], [5], [30], [31] y variables bioquímicas como: actividad enzimática [27], [31], [32], actividad enzimática de antioxidantes [27] y, concentración de proteínas y fenoles [32].

El TMG es el parámetro que ha presentado mejores y más significativos resultados en la determinación del efecto sobre la velocidad de germinación en diferentes especies vegetales de interés agrícola tratadas con campo magnético; en girasol Variedad Florasol [33], arroz [34] y en tomate [13] reportan incrementos en la velocidad de germinación de 13,83\%, 7,78\% y $3,66-8,37 \%$ respectivamente, demostrando la incidencia positiva en los procesos biológicos que se activan por la estimulación magnética durante la germinación de las semillas.
Mediante observaciones y mediciones realizadas de variables biológicas, se infiere que hay un efecto biológico reproducible. Pero, la incidencia específica de la estimulación magnética sobre los mecanismos de acción biofísicos y bioquímicos activados en semillas no ha sido establecida de manera precisa, aunque hay reportes que proponen activación de procesos como: reorientación de las moléculas con propiedades paramagnéticas en dirección del campo magnético [35], transferencia de energía del campo magnético a los tejidos cuando la frecuencia del campo es similar a la frecuencia de movimiento de resonancia del ion ciclotrón [36], influencia del campo magnético en los procesos bioquímicos que presentan radicales libres [37] y en la remoción de calcio de la membrana celular induciendo la formación de poros temporales [36] que pueden alterar la presión osmótica y la capacidad de absorción de agua [38].

El aumento en la absorción de agua por semillas estimuladas con campo magnético se ha estudiado en soja [26], maíz [6], pepino [39] y lechuga [30], [40], registrándose incrementos con respecto al control entre el $17 \%$ al $90 \%$ en estas especies. Este aumento puede ser una respuesta a un incremento en la movilidad molecular del agua [6], efecto probablemente generado por una interacción del campo magnético con las corrientes iónicas que suscitan alteraciones en la concentración iónica y presión osmótica, que a su vez provocan modificaciones en el flujo de agua a través de la membrana celular [40].

La soja es una planta de importancia agrícola con una producción mundial de 253 millones de toneladas en 2012 de la cual Colombia aportó el 0,03\% [41]. En la actualidad se investiga su utilización como fuente alterna de energía, principalmente para producir biocombustible, con un carácter competitivo en costos de producción de combustibles procedentes de los yacimientos fósiles. El maíz, la canola, el olivo y la soja son potenciales fuentes alternativas de energía, pero la soja presenta alta 
eficiencia en la emisión de energía por gramo-segundo [42], lo que hace más relevante investigar metodologías para incrementar su rendimiento mediante bioestimulación.

Con la estimulación magnética de semillas de soja se ha determinado que hay efectos significativos sobre la germinación [26], [31], [43], la absorción y mejora en procesos fotosintéticos [26], índices de vigor [26], [27], actividad enzimática [27], [31], [32], actividad enzimática de antioxidantes, masa, longitud de plántulas germinadas [27], concentración de proteínas y fenoles [32] y electroconductividad específica [31].

En este trabajo se presenta el resultado de analizar el efecto del campo magnético estático homogéneo en la germinación de soja y se sometió a prueba la hipótesis que supone que el aumento en la velocidad de germinación se debe a un incremento en la rapidez de absorción de agua en semillas sometidas a estimulación magnética.

\section{MATERIALES Y MÉTODOS}

Para realizar un correcto tratamiento magnético y buscando unificar los criterios de diseño experimental, se siguió una metodología que se obtuvo del análisis de los reportes de Valberg y Pietruszewski et al., [44], [45] y del estudio de las variables físicas involucradas, mediante la caracterización de las fuentes de campo magnético.

La estimulación se realizó con campo magnético estático homogéneo, usando dos tipos de fuentes; un electroimán con núcleos circulares de $9,0 \mathrm{~cm}$ de diámetro y separación entre estos de $6,0 \mathrm{~cm}$; y un set de imanes toroidales; ambos generando valores de 125 y 300 mT. Para determinar dónde se posicionaban las semillas en ambas fuentes, se realizó la caracterización de la distribución espacial de la densidad de flujo magnético (B) usando un teslámetro digital FW Bell 5180. Se obtuvo para el electroimán un volumen cilíndrico de
$47,0 \mathrm{~cm}^{3}$ correspondiente a un $98 \%$ de homogeneidad y para los imanes se determinó una pequeña zona circular concéntrica de homogeneidad de $88 \%$ y volumen 0,072 $\mathrm{cm}^{3}$, que corresponderán a una semilla, asumiendo que se ubican de manera concéntrica. Los niveles de los factores independientes: densidad de flujo magnético (B) y tiempo de exposición (factores fijos) utilizados en cada uno de los dos experimentos factoriales, fueron determinados a partir de la revisión realizada por los autores, así como por los tipos de imanes con que se contaba; generándose seis tratamientos o dosis diferentes como se muestra en la Tabla 1.

Tabla 1. Dosis magnéticas aplicadas relacionando campo
magnético y tiempo de exposición. Fuente: Autores
\begin{tabular}{ccc} 
& $125 \mathrm{mT}$ & $300 \mathrm{mT}$ \\
\hline $10 \mathrm{~min}$ & $\mathrm{~B}_{11}$ & $\mathrm{~B}_{13}$ \\
$20 \mathrm{~min}$ & $\mathrm{~B}_{21}$ & $\mathrm{~B}_{23}$ \\
$60 \mathrm{~min}$ & $\mathrm{~B}_{61}$ & $\mathrm{~B}_{63}$ \\
\hline
\end{tabular}

La estimulación con el electroimán y el set de imanes toroidales se realizó con las mismas dosis.

Las semillas de soja presentaban uniformidad en sus características. La siembra se realizó en cajas de Petri una vez terminada la estimulación magnética; con cuatro repeticiones de veinticinco semillas por tratamiento. El proceso de germinación de las semillas se efectuó en condiciones de laboratorio, con temperatura de $19^{\circ} \mathrm{C} \pm 1^{\circ} \mathrm{C}$, humedad relativa de $18 \%$ y fotoperiodo 16 8 horas. Para los dos experimentos factoriales se usó un grupo de semillas sin exposición magnética como control. Se asumieron como semillas germinadas aquellas cuya radícula tuviera una longitud de un milímetro tras romper la testa. La medida de la absorción de agua se realizó cada hora durante las siete primeras horas después de estimuladas las semillas, para esto se depositaron en medio de algodón con 50 $\mathrm{ml}$ de agua. La masa fue medida con una balanza digital SHIMADZU con resolución $0,01 \mathrm{~g}$. 
Para el análisis de los resultados de germinación se usó el programa Seedcalculator teniendo en cuenta los parámetros TMG, Gmax (germinación máxima) y los tiempos de germinación (T1, T10, T25, T50, T75 у T90).

La elección de la prueba estadística para el análisis de la absorción de agua se rigió bajo la evaluación de dos supuestos del diseño completamente aleatorizado: distribución normal de los errores (Shapiro-wilks) y homocedasticidad (Bartlett). Dada la naturaleza no paramétrica de los datos, se realizó la prueba de KruskalWallis con el programa R-3.1.1.

\section{RESULTADOS Y DISCUSIONES}

\subsection{Germinación}

Se presentan los resultados experimentales usando el electroimán como fuente magnética dado que estos tuvieron mejores respuestas que los arrojados por los imanes toroidales. De los parámetros analizados para los diferentes tratamientos respecto al control, el TMG presentó las mejores respuestas, registrándose para las dosis $\mathrm{B}_{13}$ diferencias muy fuertemente significativas (ver Tabla 2), $\mathrm{B}_{61}$ diferencias significativas (disminuyó $10,59 \%$ con respecto al control que corresponde a 6,48 horas) (Fig. 1), y $\mathrm{B}_{63}$ y $\mathrm{B}_{23}$ diferencias (reducciones de $7,06 \%$ correspondiendo a 4,32 horas $y$ $4,71 \%$ equivalente 2,88 horas con relación al control respectivamente). En la Tabla 2 se puede observar que para $\mathrm{B}_{13}$ el TMG disminuyó en $17,65 \%$ equivalente a $10,80 \mathrm{~h}$ respecto al control, congruente con el reporte de Shine que presenta incrementos en la velocidad de germinación hasta del $42 \%$ para dosis de $200 \mathrm{mT} / 60 \mathrm{~min}$ [26]. Por otro lado, $\mathrm{B}_{13}$ no exhibió diferencias significativas con la dosis $\mathrm{B}_{61} \mathrm{y}$ el tratamiento $\mathrm{B}_{21}$. Presentando este último el TMG más prolongado (28,8 minutos más que el control) en comparación con las dosis $\mathrm{B}_{13}$ y $\mathrm{B}_{61}$, como se muestra en la Tabla 2.

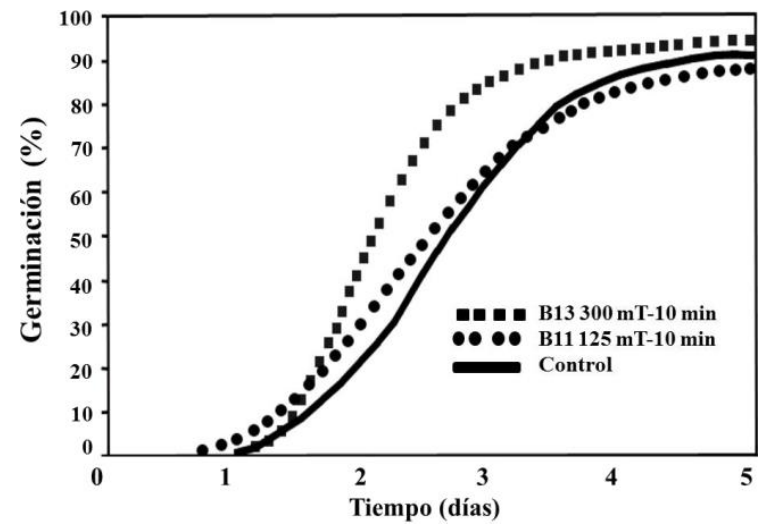

Fig. 1. Curva de germinación de semillas de soja estimuladas magnéticamente con electroimán. Fuente: Autores

El parámetro Gmax registró un incremento de 5,0\% en la germinación promedio para el tratamiento $\mathrm{B}_{61}$ (Tabla 2). Sin embargo, estadísticamente no presentó diferencias significativas con respecto al control (P-value 0,75), ni entre las dosis de exposición magnética, estableciendo que el tratamiento magnético no tuvo una influencia de forma relevante en el total de semillas germinadas, coincidiendo con el reporte de Shine y colaboradores [26] quienes exponen que los tratamientos magnéticos no inciden de manera significativa en el porcentaje de germinación de semillas estimuladas para diferentes dosis entre 50 $\mathrm{mT}$ a $300 \mathrm{mT}$ en pasos de $50 \mathrm{mT}$ para 30 , 60 y $90 \mathrm{~min}$, y discrepando con los resultados de Camps-Raga, que reportan incrementos significativos en la germinación de $10,64 \%$ en semillas de soja tratadas a 0,94 mT-6 horas [43].

Los tiempos para que germinaran el $10 \%, 25 \%, 50 \%$ y $75 \%$ de las semillas, presentaron diferencias significativas respecto al control. La dosis $\mathrm{B}_{13}$ registró disminución de 22,89\% (18,24 horas), $21,46 \%$ (23,44 horas), $16,42 \%$ (7,92 horas) y $8,44 \%$ (3,12 horas) para los tiempos T75, T50, T25 y $\mathrm{T} 10$, respectivamente. Por otra parte las dosis $\mathrm{B}_{11}$ y $\mathrm{B}_{23}$ disminuyeron el tiempo $\mathrm{T} 10$ en $17,53 \%(6,48$ horas) y $10,39 \%(3,84$ horas) respectivamente. El tiempo T75 presentó reducción de $15,96 \%(12,72$ horas) y $10,24 \%$ (8,16 horas) con las dosis $\mathrm{B}_{61}$ y $\mathrm{B}_{63}$ respectivamente. 
Efecto del campo magnético estático homogéneo en la germinación y absorción de agua en semillas de soja

Tabla 2. Parámetros de germinación expresados en media y error estándar para semillas de soja expuestas a campo magnético estático homogéneo usando electroimán. Fuente: Autores

\begin{tabular}{|c|c|c|c|c|c|c|c|}
\hline $\begin{array}{l}\text { Parámetros de } \\
\text { germinación }\end{array}$ & $\mathrm{B}_{11}$ & $\mathrm{~B}_{13}$ & $\mathrm{~B}_{21}$ & $\mathrm{~B}_{23}$ & $\mathrm{~B}_{61}$ & $\mathrm{~B}_{63}$ & Control \\
\hline Gmáx (\%) & $90 \pm 3,46$ & $93 \pm 1,00$ & $93 \pm 1,91$ & $93 \pm 1,91$ & $96 \pm 2,83$ & $93 \pm 1,91$ & $91 \pm 3,42$ \\
\hline TMG (días) & $2,42 \pm 0,09$ & $2,10 \pm 0,03^{* * * *}$ & $2,57 \pm 0,12$ & $2,43 \pm 0,04^{*}$ & $2,28 \pm 0,10^{* *}$ & $2,37 \pm 0,04^{*}$ & $2,55 \pm 0,04$ \\
\hline $\mathrm{T} 1$ (días) & $0,65 \pm 0,08$ & $1,02 \pm 0,06$ & $0,98 \pm 0,14$ & $0,73 \pm 0,12$ & $0,91 \pm 0,04$ & $0,88 \pm 0,09$ & $0,86 \pm 0,08$ \\
\hline T10 (días) & $1,27 \pm 0,11^{* *}$ & $1,41 \pm 0,02^{* * *}$ & $1,64 \pm 0,14$ & $1,38 \pm 0,04^{* *}$ & $1,43 \pm 0,10$ & $1,49 \pm 0,10$ & $1,54 \pm 0,02$ \\
\hline T25 (días) & $1,73 \pm 0,12^{*}$ & $1,68 \pm 0,02^{* * * *}$ & $2,08 \pm 0,13$ & $1,86 \pm 0,07^{*}$ & $1,79 \pm 0,13$ & $1,91 \pm 0,09$ & $2,01 \pm 0,03$ \\
\hline T50 (días) & $2,32 \pm 0,12^{*}$ & $2,05 \pm 0,03^{* * * *}$ & $2,62 \pm 0,12$ & $2,46 \pm 0,11$ & $2,25 \pm 0,15^{*}$ & $2,41 \pm 0,08^{*}$ & $2,61 \pm 0,06$ \\
\hline T75 (días) & $3,00 \pm 0,11^{*}$ & $2,56 \pm 0,03^{* * * *}$ & $3,22 \pm 0,09$ & $3,16 \pm 0,03$ & $2,79 \pm 0,18^{* *}$ & $2,98 \pm 0,04^{* *}$ & $3,32 \pm 0,09$ \\
\hline T90 (días) & $3,70 \pm 0,12$ & $3,60 \pm 0,25$ & $4,01 \pm 0,98$ & $4,15 \pm 1,00$ & $3,46 \pm 0,79$ & $3,76 \pm 0,93$ & $4,66 \pm 1,45$ \\
\hline
\end{tabular}

Gmáx: número de semillas germinadas (\%); TMG: tiempo medio de germinación (días); T1, T10, T25, T50, T75, T90: tiempo en días requerido para que germine el $1,10,25,50,75$ y $90 \%$ de las semillas. El asterisco indica diferencias con el control: **** $(\mathrm{P}<0,001)$ muy fuertemente significativo, ${ }^{* * *}(0,001<\mathrm{P}<0,01)$ fuertemente significativo, ${ }^{* *}(0,01<\mathrm{P}<0,05)$ significativo $\mathrm{y} *$ $(0,05<\mathrm{P}<0,1)$ diferencias

Disminuciones de estos mismos parámetros fueron obtenidas en arroz [34], tomate [13], Festuca arundinacea [46] y Grass [47], aunque los resultados de estas investigaciones se registraron con densidades de 125 y $250 \mathrm{mT}$, los tiempos de exposición fueron superiores a los del presente estudio.

El descenso en el tiempo de germinación de diferentes semillas puede ser desencadenado por moléculas con propiedades magnéticas que atraen y transfieren la energía del campo magnético a otras estructuras celulares que son activadas [48], permitiendo incremento en la actividad de enzimas hidrolíticas como la a-amilasa que está involucrada en el proceso metabólico durante la germinación.

Los tiempos T1 y T90 correspondientes al inicio y final de la germinación no fueron alterados por la estimulación magnética, respecto al control. Para T1 se estableció que el inicio de la latencia no presenta alteraciones percibidas en la germinación, sino hasta el final de este periodo (T10), en el que se registraron los primeros efectos significativos por la estimulación magnética. El tiempo para que germinara el $90 \%$ no presentó diferencias estadísticas con respecto al control, determinando que el
T90 es el tiempo máximo de germinación para todos los tratamientos y a su vez el $90 \%$ es el porcentaje de germinación máximo para todas las dosis. Los resultados obtenidos con $\mathrm{T} 1$ permiten establecer un límite de tiempo de detección del efecto de la estimulación magnética para el análisis de la germinación, que podría ser característico de la especie estudiada y de la dosis de exposición magnética bajo condiciones experimentales establecidas. Este límite mínimo de tiempo de detección es dependiente tanto de los procesos metabólicos como biológicos suscitados en la superación de la dormancia, donde la inducción magnética tendría puntos de acción tal como en el desdoblamiento de reservas energéticas donde se incrementa la actividad enzimática y se tienen reportes sobre el efecto en el incremento de la concentración de enzimas como la alfa-amilasa por estimulación magnética [6], [39], [49], [50].

\subsection{Absorción de agua}

En la Tabla 3 los resultados de la absorción de agua y el delta de la absorción de agua, establecen que para los tratamientos magnéticos $\mathrm{B}_{11}, \mathrm{~B}_{13}$ y $\mathrm{B}_{23}$ hay una tendencia a incrementar la capacidad de 
absorción de agua durante las primeras 6 horas (Fig. 2), Pero no existen diferencias estadísticas respecto al grupo control ( $\mathrm{P}$ values 0,578 y 0,986 , Tabla 3 ). Esto permite inferir que las dosis evaluadas en esta investigación, no tuvieron la suficiente eficiencia para generar el incremento en el tamaño de los poros de la capa de las semillas, como factor favorable en el incremento de la absorción de agua en semillas [51].

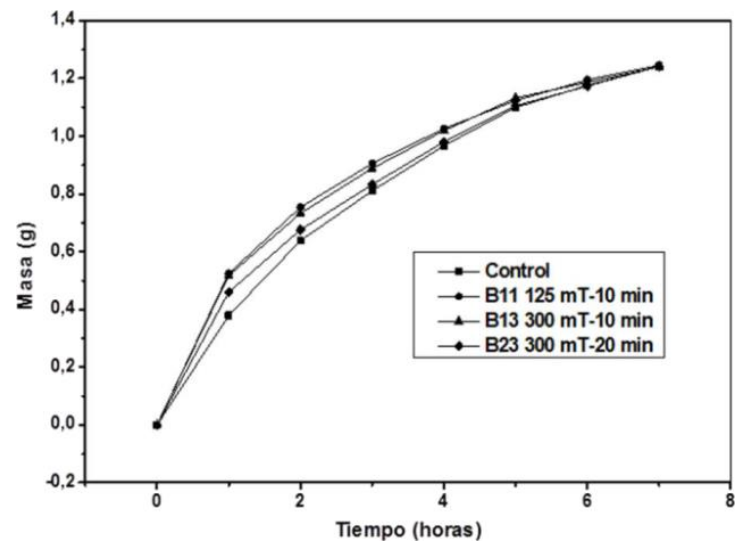

Fig. 2. Comportamiento de absorción de agua en función del tiempo para los tratamientos magnéticos y control. Fuente: Autores

\section{CONCLUSIONES}

Los resultados obtenidos permiten inferir que la interacción del campo magnético con la materia, es decir con las semillas, activa los mecanismos que desencadenan respuestas biológicas en semillas de soja y esta activación se da bajo valores determinados de inducción magnética y tiempo de exposición. En este estudio, la germinación de semillas de soja fue mejorada para los parámetros velocidad de germinación y germinación máxima, con los tratamientos de $300 \mathrm{mT}$ - 10 min comparado con el control, pero se debe plantear la continuidad experimental para llevar este tipo de estimulación física a condiciones de campo.

Se evidencia que los mecanismos de activación biológica afectados por el tratamiento magnético se hacen notorios después de $30 \mathrm{~h}$ de la siembra, lo que plantea que si se desea realizar pruebas químicas a las semillas estimuladas, estas se deben realizar después de este tiempo.

Dado que se observa un aumento en la rapidez de absorción de agua con dos dosis, pero que no presentan diferencias estadísticamente significativas en este proceso, no es descartable por completo la hipótesis que relaciona el aumento en la rapidez de germinación con la aceleración en la absorción de agua. Lo que plantea la necesidad de seguir estudiando esta hipótesis con este tipo de semilla y/o con otras.

Tabla 3. Valores de absorción de agua de semillas de soja expuestas a campo magnético estático homogéneo.

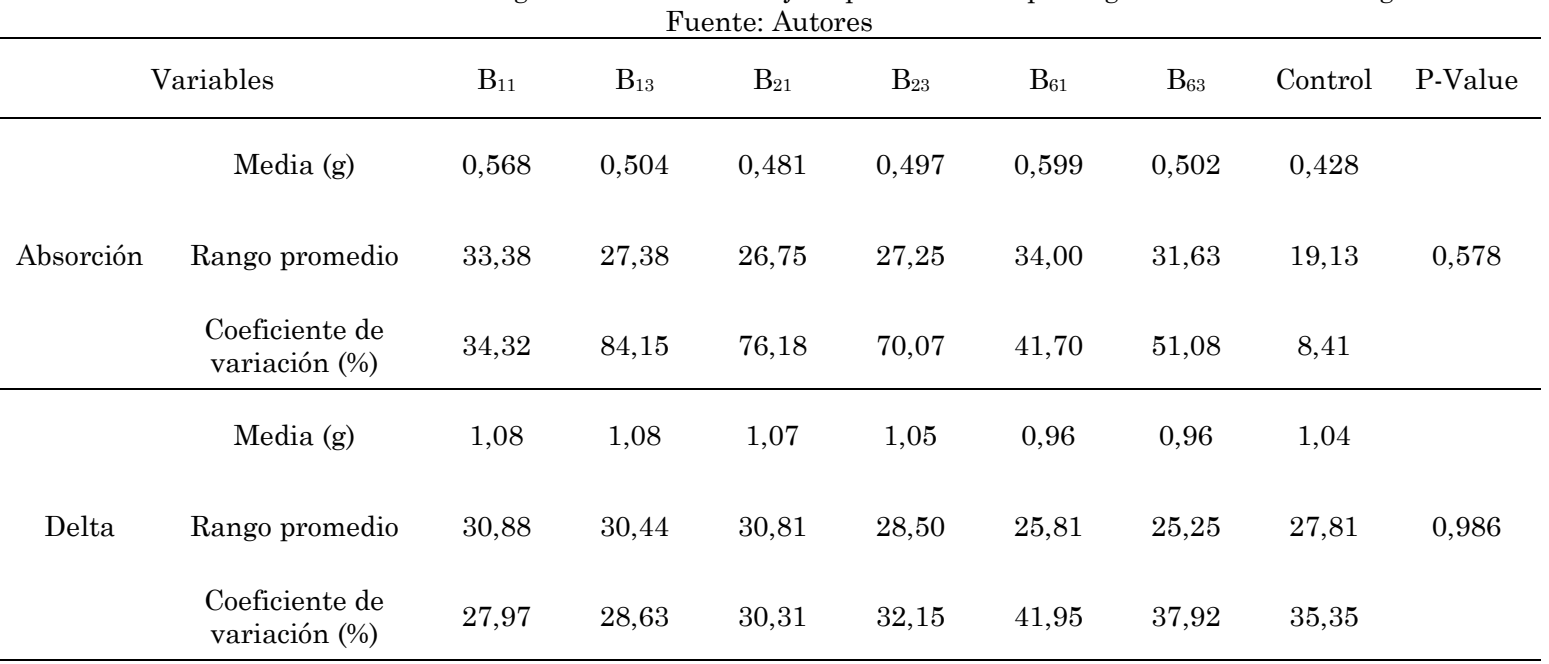

Para la absorción de agua no se registraron diferencias significativas 


\section{AGRADECIMIENTOS}

Este trabajo contó con el apoyo de la Vicerrectoría de Investigación de la Universidad de Caldas (proyecto código VIP 0084311), el Grupo de Investigación en Bioelectromagnetismo Aplicado a la Ingeniería Agroforestal adscrito a la Universidad Politécnica de Madrid, España y el programa Jóvenes Investigadores de Colciencias.

\section{REFERENCIAS}

[1] P. Galland and A. Pazur, "Magnetoreception in plants," J. Plant Res., vol. 118, no. 6, pp. 371389, Dec. 2005.

[2] A. Aladjadjiyan, "Study of the influence of magnetic field on some biological characteristics of Zea mais," J. Cent. Eur. Agric., vol. 3, no. 2, pp. 89-94, 2002.

[3] M. Flórez, M. V. Carbonell, and E. Martínez, "Exposure of maize seeds to stationary magnetic fields: Effects on germination and early growth," Environ. Exp. Bot., vol. 59, no. 1, pp. 68-75, Jan. 2007.

[4] A. Vashisth and S. Nagarajan, "Germination Characteristics of Seeds of Maize (Zea mays L .) Exposed to Magnetic Fields under Accelerated Ageing Condition," J. Agric. Phys., vol. 9, pp. 50$58,2009$.

[5] A. Vashisth and S. Nagarajan, "Characterization of water binding and germination traits of magnetically exposed maize (Zea mays L.) seeds equilibrated at different relative humidities at two temperatures," Indian J. Biochem. Biophys., vol. 46, no. 2, pp. 184-191, Apr. 2009.

[6] A. Vashisth and S. Nagarajan, "Characterization of water distribution and activities of enzymes during germination in magnetically-exposed maize (Zea mays L) seeds.," Indian J. Biochem. Biophys., vol. 47, no. 5, pp. 311-318, Oct. 2010.

[7] R. Zepeda-Bautista, A. Hernández-Aguilar, C Domínguez-Pacheco, A. Cruz-Orea, J. GodinaNava, and E. Martínez-Ortíz, "Electromagnetic field and seed vigour of corn hybrids," Int. Agrophysics, vol. 24, no. 2007, pp. 329-332, 2010.

[8] A. Domínguez Pacheco, C. Hernández Aguilar, A. Cruz Orea, A. Carballo Carballo, R. Zepeda Bautista, and E. M. Martínez Ortíz, "Semilla de maíz bajo la influencia de irradiación de campos electromagnéticos," Rev. Fitotec. Mex., vol. 33, no. 2, pp. 183-188, 2010.

[9] E. Isaac, C. Hernández, A. Domínguez, and A. Cruz, "Effect of pre-sowing electromagnetic treatment on seed germination and seedling growth in maize (Zea mays L.)," Agron. Colomb., vol. 29, no. 2, pp. 213-220, 2011.

[10] R. B. Zepeda, C. A. Hernández, F. L. Suazo, A. P. Domínguez, A. O. Cruz, E. O. Martínez, and L. M. S. Hernández, "Physical characteristics of maize grain and tortilla exposed to electromagnetic field," Int. Agrophysics, vol. 25, pp. 389-393, 2011.

[11]D. G. Fernández, A. S. Torres, and L. S. Pelegrín, "Efecto del tratamiento magnético de semillas de cebolla (Allium cepa L.) sobre la germinación y el crecimiento de las plántulas," Alimentaria, vol. 337, pp. 181-186, 2002.

[12] A. De Souza, D. García, L. Sueiro, L. Licea, and E. Porras, "Pre-sowing magnetic treatment of tomato seeds: effects on the growth and yield of plants cultivated late in the season," Spanish J. Agric. Res., vol. 3, no. 1, pp. 113-122, 2005.

[13] E. Martínez, M. V. Carbonell, M. Flórez, J. M. Amaya, and R. Maqueda, "Germination of tomato seeds (Lycopersicon esculentum L) under magnetic field," Int. Agrophysics, vol. 23, no. 1, pp. 45-49, 2009.

[14]A. El-Yazied, O. Shalaby, A. El-Gizawy, S. Khalf, and A. El-Satar, "Effect of magnetic field on seed germination and transplant growth of tomato," J. Am. Sci., vol. 7, no. 12 , pp. 306-312, 2011.

[15]H. Feizi, H. Sahabi, R. Parviz, N. Shahtahmassebi, O. Gallehgir, and $\mathrm{S}$. Amirmoradi, "Impact of Intensity and Exposure Duration of Magnetic Field on Seed Germination of Tomato (Lycopersicon esculentum L.)," Not. Sci. Biol., vol. 4, no. 1, pp. 116-120, 2012.

[16] T. Gemishev, K. Tsolova, and M. Markov, "Static Magnetic Field Influence on the Activity of some Respiratory Enzymes in Wheat," Electromagn. Biol. Med., vol. 13, no. 2, pp. 107-115, Jan. 1994.

[17]S. Wojcik, "Effect of the pre-sowing magnetic biostimulation of the buckwheat seeds on the yield and chemical composition of buckwheat grain," Curr. Adv. Buckwheat Res, pp. 667-674, 1995.

[18]S. Pietruszewski, "Effects of magnetic biostimulation of wheat seeds on germination, yield and proteins," Int. Agrophysics, vol. 10, pp. 51-55, 1996.

[19]A. Gholami, S. Sharafi, and H. Abbasdokht, "Effect of Magnetic Field on Seed Germination of Two Wheat Cultivars," World Acad. Sci. Eng. Technol., vol. 4, no. 8, pp. 1385-1387, 2010.

[20] J. Podleœny and L. Misiak, "Concentration of free radicals in pea seeds after pre-sowing treatment with magnetic field," Int. Agrophysics, vol. 19, no. 3, pp. 243-249, 2005.

[21] J. Podleœny, S. Pietruszewski, and A. Podleœna, "Influence of magnetic stimulation of seeds on the formation of morphological features and yielding of the pea," Int. Agrophysics, vol. 19, no. 1, pp. 61-68, 2005. 
[22] E. Martínez, M. Flórez, R. Maqueda, M. V Carbonell, and J. M. Amaya, "Pea (Pisum sativum, L.) and lentil (Lens culinaris, Medik) growth stimulation due to exposure to 125 and 250 mT stationary fields," Polish J. Environ. Stud., vol. 18, no. 4, pp. 657-663, 2009.

[23] M. V Carbonell, M. Florez, E. Martínez, R. Maqueda, and J. M. Amaya, "Study of stationary magnetic fields on initial growth of pea (Pisum sativum L.) seeds," Seed Sci. Technol., vol. 39, no. 3, pp. 673-679, 2011.

[24] C.. Atak, Ö. Emiro, S. Alikamano, and A. Rzakoulieva, "Stimulation of regeneration by magnetic field in soybean (Glycine max L . Merrill ) tissue cultures," J. Cell Mol. Biol., vol. 2, pp. 113-119, 2003.

[25] Ç. Atak, Ö. Çelik, A. Olgun, S. Alikamanoğlu, and A. Rzakoulieva, "Effect of Magnetic Field on Peroxidase Activities of Soybean Tissue Culture," Biotechnol. Biotechnol. Equip., vol. 21, no. 2, pp. 166-171, Jan. 2014.

[26] M. B. Shine, K. N. Guruprasad, and A. Anand, "Enhancement of germination, growth, and photosynthesis in soybean by pre-treatment of seeds with magnetic field," Bioelectromagnetics, vol. 32, no. 6, pp. 474-484, Sep. 2011.

[27] M. B. Shine, K. N. Guruprasad, and A. Anand, "Effect of stationary magnetic field strengths of 150 and $200 \mathrm{mT}$ on reactive oxygen species production in soybean.," Bioelectromagnetics, vol. 33, no. 5, pp. 428-37, Jul. 2012.

[28] M. Flórez, E. Martínez, M. Carbonell, and M. Flórez, "Effect of Magnetic Field Treatment on Germination of Medicinal Plants Salvia officinalis L. and Calendula officinalis L.," Polish J. Environ. Stud., vol. 21, no. 1, pp. 57-63, 2012.

[29] M. Iqbal, Z. Haq, Y. Jamil, and M. Ahmad, "Effect of presowing magnetic treatment on properties of pea," Int. Agrophysics, vol. 26, no. 1, pp. 25-31, Jan. 2012.

[30] F. G. Reina, L. A. Pascual, and I. A. Fundora, "Influence of a stationary magnetic field on water relations in lettuce seeds. Part II: Experimental results," Bioelectromagnetics, vol. 22, no. 8, pp. 596-602, Dec. 2001.

[31]A. Aladjadjiyan, "Use of physical factors as an alternative to chemical amelioration," $J$. Environ. Prot. Ecol, vol. 4, no. 3, pp. 662-667, 2003.

[32] R. Radhakrishnan, T. Leelapriya, and B. D. R. Kumari, "Effects of pulsed magnetic field treatment of soybean seeds on calli growth, cell damage, and biochemical changes under salt stress," Bioelectromagnetics, vol. 33, no. 8, pp. 670-681, Dec. 2012.

[33] M. Carbonell, E. Martínez, M. Flórez, and J. Amaya, "Influencia de campos magnéticos estacionarios de $125 \mathrm{mT}$ y $250 \mathrm{mT}$ en la germinación de semillas de girasol," Ing. Recur. Nat. y del Ambient., vol. 3, pp. 34-39, 2005.
[34]M. Flórez, M. V. Carbonell, and E. Martínez, "Early Sprouting and First Stages of Growth of Rice Seeds Exposed to a Magnetic Field," Electromagn. Biol. Med., vol. 23, no. 2, pp. 157 166, Jan. 2004.

[35]A. Aladjadjiyan and A. Zahariev, "Influence of Stationary Magnetic Field on the Absorption Spectra of the Photosynthetic Apparatus of Some Ornamental Perennial Species," Bulg. J. Phys, vol. 29, no. 3-4, pp. 179-183, 2002.

[36] A. Majd, A. Shabrangi, M. Bahar, and S. Abdi, "Effect of AC and DC Magnetic Fields on Seed Germination and early vegetative growth in Brassica Napus L," Prog. Electromagn. Res. Symp. Proc., pp. 710-714, 2009.

[37] F. Dhawi, J. M. Al-khayri, and E. Hassan, "Static Magnetic Field Influence on Elements Composition in Date Palm (Phoenix dactylifera L.)," Res. J. Agric. Biol. Sci., vol. 5, no. 2, pp. 161-166, 2009.

[38]A. Calatayud, D. J. Iglesias, M. Talón, and E. Barreno, "Effects of 2-month ozone exposure in spinach leaves on photosynthesis, antioxidant systems and lipid peroxidation," Plant Physiol. Biochem., vol. 41, no. 9, pp. 839-845, Sep. 2003.

[39] J. Bhardwaj, A. Anand, and S. Nagarajan, "Biochemical and biophysical changes associated with magnetopriming in germinating cucumber seeds," Plant Physiol. Biochem., vol. 57, pp. 6773, Aug. 2012.

[40] F. G. Reina and L. A. Pascual, "Influence of a stationary magnetic field on water relations in lettuce seeds. Part I: Theoretical considerations," Bioelectromagnetics, vol. 22, no. 8, pp. 589-595, Dec. 2001.

[41]FAO, "FAOSTAT," Food and Agriculture Organization of the United Nations, [Online]. Available: http://faostat3.fao.org, [Accessed: 15Mar-2014].

[42] M. Giampietro, K. Mayumi, and J. RamosMartin, "Can Biofuels Replace Fossil Energy Fuels? A Multi-Scale Integrated Analysis Based on the Concept of Societal and Ecosystem Metabolism: Part 1," Int. J. Transdiscipl. Res., vol. 1, no. 1, pp. 51-87, 2006.

[43] B. Camps-Raga, S. Gyawali, and N. E. Islam, "Germination rate studies of soybean under static and low-frequency magnetic fields," IEEE Trans. Dielectr. Electr. Insul., vol. 16, no. 5, pp. 1317-1321, 2009.

[44] W. T. Kaune, "Designing EMF experiments: what is required to characterize 'exposure'?," Bioelectromagnetics, vol. 16, no. 6, pp. 402-404, 1995.

[45] S. Pietruszewski, S. Muszyñski, and A. Dziwulska, "Electromagnetic fields and electromagnetic radiation as non-invasive external stimulants for seeds (selected methods and responses)," Int. Agrophysics, vol. 21, no. 1, pp. 95-100, 2007. 
[46] M. V. Carbonnel, E. Martínez, M. Flórez, R. Maqueda, A. Pintor-LÓPEZ, and J. M. Amaya, "Magnetic field treatments improve germination and seedling growth in Festuca arundinacea Schreb. and Lolium perenne L.," Seed Sci. Technol., vol. 36, no. 1, pp. 31-37, Apr. 2008.

[47] M. F. García, E. M. Ramírez, and M. V. C. Padrino, "Germination of grass seeds subjected to stationary magnetic field," Ing. Recur. Nat. y del Ambient., vol. 7, pp. 12-17, 2008.

[48]A. Aladjadjiyan, "Influence of stationary magnetic field on lentil seeds," Int Agrophysics, vol. 24 , no. 3 , pp. 321-324, 2010 .
[49]A. Vashisth and S. Nagarajan, "Effect on germination and early growth characteristics in sunflower (Helianthus annuus) seeds exposed to static magnetic field," J. Plant Physiol., vol. 167, no. 2, pp. 149-156, Jan. 2010.

[50] I. Afzal, K. Mukhtar, M. Qasim, S. M. A. Basra, M. Shahid, and Z. Haq, "Magnetic stimulation of marigold seed," Int. Agrophysics, vol. 26, no. 4, pp. 335-339, Jan. 2012.

[51] A. K. Smith, A. M. Nash, and L. I. Wilson, "Water absorption of soybeans," J. Am. Oil Chem. Soc., vol. 38, no. 3, pp. 120-123, Mar. 1961. 